\title{
Positive Solutions to a Coupled Fractional Differential System with $p$-Laplacian Operator
}

\author{
Yuehan Liu, Xiaodi Zhao, and Huihui Pang \\ College of Science, China Agricultural University, Beijing 100083, China \\ Correspondence should be addressed to Huihui Pang; phh2000@163.com
}

Received 4 April 2019; Accepted 26 May 2019; Published 17 June 2019

Academic Editor: Douglas R. Anderson

Copyright (C) 2019 Yuehan Liu et al. This is an open access article distributed under the Creative Commons Attribution License, which permits unrestricted use, distribution, and reproduction in any medium, provided the original work is properly cited.

In this paper, we consider a fractional differential system with multistrip and multipoint mixed boundary conditions involving $p$ Laplacian operator and fractional derivatives. The existence result of positive solutions is established by the Leggett-Williams fixed point theorem. Also, an example is presented to illustrate our main result.

\section{Introduction}

Fractional calculus is a significant branch of mathematical analysis which concerns derivatives and integrals. Fractional differential equations are caused by the intensive development of the theory of fractional calculus and have gained great interest currently, because of their crucial applications in various fields of physics, biology, and engineering such as control, porous media, electromagnetic, and other fields [14].

Moreover, many mathematical formulations of physical and biological phenomena lead to fractional differential equations with $p$-Laplacian operator, such as memory, fluid dynamics, predator harvesting, and hereditary properties of various materials, which are difficult to achieve in classical integer-order models. Hence, in recent years, more and more researchers have considered the fractional differential equations with $p$-Laplacian operator, by applying various mathematical theory and tools; refer to [5-9].

For example, authors in [10] discussed the following differential system with $p$-Laplacian operator

$$
\begin{aligned}
& D^{\beta_{1}} \phi_{p}\left(\left(D^{\alpha_{1}} \mu(x)\right)\right)+\Phi_{1}(x, v(x))=0, \quad x \in(0,1), \\
& D^{\beta_{2}} \phi_{p}\left(\left(D^{\alpha_{2}} v(x)\right)\right)+\Phi_{2}(x, \mu(x))=0, \quad x \in(0,1), \\
& \left.D^{\alpha_{1}} \mu(x)\right|_{x=0}=0=\left.\mu(x)\right|_{x=0}=\left.\mu^{\prime \prime}(x)\right|_{x=0}, \\
& \left.\mu^{\prime}(x)\right|_{x=1}=\eta_{1} I_{P}^{\gamma_{1}} \psi_{1}(\mu)
\end{aligned}
$$

$$
\begin{gathered}
=\frac{\eta_{1}}{\Gamma\left(\gamma_{1}\right)} \int_{0}^{P}(P-\tau)^{\gamma_{1}-1} \psi_{1}(\mu(\tau)) d \tau, \\
\left.D^{\alpha_{2}} v(x)\right|_{x=0}=0=\left.v(x)\right|_{x=0}=\left.v^{\prime \prime}(x)\right|_{x=0}, \\
\left.v^{\prime}(x)\right|_{x=1}=\eta_{2} I_{P}^{\gamma_{2}} \psi_{2}(v) \\
=\frac{\eta_{2}}{\Gamma\left(\gamma_{2}\right)} \int_{0}^{P}(P-\tau)^{\gamma_{2}-1} \psi_{2}(\mu(\tau)) d \tau,
\end{gathered}
$$

where $2<\alpha_{i}<3,0<\beta_{i}<1, P, \eta_{i}, \gamma_{i}>0, \psi_{1}, \psi_{2} \in L[0,1]$, $\phi_{p}(s)$ is a $p$-Laplacian operator: $\phi_{p}(s)=|s|^{p-2} s, p>1$ and $\Phi_{i}:[0,1] \times[0, \infty) \longrightarrow(0, \infty)$. Authors established the conclusion of the existence and uniqueness of solution to the above problem by using topological degree theory.

By reading the existing literatures [11-24], we note that the fractional differential system involving $p$-Laplacian operator and multistrip and multipoint boundary conditions has not been studied yet. Thus, in this paper, we first pay close attention to the following fractional differential system, involving $p$-Laplacian operator and lower fractional derivatives:

$$
\begin{aligned}
& D_{0^{+}}^{\beta}\left(\varphi_{p}\left(D_{0^{+}}^{\alpha} u(t)\right)\right) \\
& \quad=f_{1}\left(t, u(t), v(t), D_{0^{+}}^{\alpha} u(t), D_{0^{+}}^{\alpha} v(t)\right), \\
& D_{0^{+}}^{\beta}\left(\varphi_{p}\left(D_{0^{+}}^{\alpha} v(t)\right)\right) \\
& \quad=f_{2}\left(t, u(t), v(t), D_{0^{+}}^{\alpha} u(t), D_{0^{+}}^{\alpha} v(t)\right),
\end{aligned}
$$


with multistrip and multipoint boundary conditions:

$$
\begin{aligned}
u(0) & =D_{0^{+}}^{\alpha} u(0)=0 \\
u(1) & =\sum_{i=1}^{m} b_{i} \int_{0}^{\xi_{i}} u(t) d A(t), \\
\varphi_{p}\left(D_{0^{+}}^{\alpha} u(1)\right) & =\sum_{i=1}^{m} a_{i} \varphi_{p}\left(D_{0^{+}}^{\alpha} u\left(\xi_{i}\right)\right), \\
v(0) & =D_{0^{+}}^{\alpha} v(0)=0 \\
v(1) & =\sum_{i=1}^{m} d_{i} \int_{0}^{\eta_{i}} v(t) d B(t), \\
\varphi_{p}\left(D_{0^{+}}^{\alpha} v(1)\right) & =\sum_{i=1}^{m} c_{i} \varphi_{p}\left(D_{0^{+}}^{\alpha} v\left(\eta_{i}\right)\right),
\end{aligned}
$$

where $D_{0+}^{\alpha}$ and $D_{0+}^{\beta}$ are the Riemann-Liouville fractional derivatives of order $\alpha$ and $\beta, f:[0,1] \times \mathbb{R}^{+2} \times \mathbb{R}^{2} \longrightarrow \mathbb{R}^{+}$ is a continuous function. For $i=1,2, \ldots, m, 1<\alpha \leqslant 2$, $1<\beta \leqslant 2,0<\xi_{i} \leq 1,0<\eta_{i} \leq 1, a_{i}, b_{i}, c_{i}, d_{i}$ are nonnegative constants. $\varphi_{p}(s)=|s|^{p-2} s, p>1, \varphi_{p}^{-1}=\varphi_{q}, 1 / p+1 / q=$ 1. And $A(t), B(t)$ are nondecreasing functions of bounded variation in $[0,1]$.

We emphasize that, despite the complication of the discrete and integral boundary conditions (3) and (4), operator $\mathrm{T}$ can be presented in a concise form based on the piecewise form of Green's function. And we can accurately estimate the upper and lower bounds of its value, which is fully prepared for the establishment of the main theorem.

Accordingly, the conclusion we reached is an extensive result and a meaningful supplement to the theory of the fractional differential systems with $p$-Laplacian operator.

Theorem 1 (the Leggett-Williams fixed point theorem). Let $P$ be a cone in a real Banach space $E, P_{c}=\{x \in P \mid\|x\| \leqslant c\}, \Psi$ be a nonnegative continuous concave function on $P$ such that $\Psi(x) \leqslant\|x\|$ for all $x \in \bar{P}_{c}$, and $P(\Psi, b, d)=\{x \in P \mid b \leqslant$ $\Psi(x),\|x\| \leqslant d\}$. Suppose that $T: \bar{P}_{c} \longrightarrow \bar{P}_{c}$ is completely continuous and there exist constants $0<a<b<d \leqslant c$ such that

$$
\begin{aligned}
& \left(S_{1}\right) \quad\{x \in P(\Psi, b, d) \mid \Psi(x)>b\} \neq \varnothing, \text { and } \Psi(T x)> \\
& \quad b \text { for } x \in P(\Psi, b, d) \\
& \left(S_{2}\right)\|T x\|<a \text { for }\|x\| \leqslant a ; \\
& \left(S_{3}\right) \Psi(T x)>b \text { for } x \in P(\Psi, b, c), \text { with }\|T x\|>d .
\end{aligned}
$$

Then $T$ has at least three fixed points $x_{1}, x_{2}$, and $x_{3}$ which satisfy

$$
\begin{aligned}
\left\|x_{1}\right\| & <a, \\
b & <\Psi\left(x_{2}\right), \\
a & <\left\|x_{3}\right\|
\end{aligned}
$$

$$
\text { with } \Psi\left(x_{1}\right)<b \text {. }
$$

\section{Preliminaries}

In this section, we will put forward some crucial definitions and theorems, preparing for establishing the existence of solutions.

Definition 2. The Riemann-Liouville fractional integral of order $\alpha>0$ of a function $f:(0, \infty) \longrightarrow \mathbb{R}$ is given by

$$
I_{0+}^{\alpha} f(t)=\frac{1}{\Gamma(\alpha)} \int_{0}^{t}(t-s)^{\alpha-1} f(s) d s
$$

provided the right-hand side is pointwise defined on $(0, \infty)$, where $\Gamma(\alpha)$ is the Euler gamma function defined by $\Gamma(\alpha)=$ $\int_{0}^{\infty} t^{\alpha-1} e^{-t} d t$, for $\alpha>0$.

Definition 3. The Riemann-Liouville fractional derivative of order $\alpha>0$ for a function $f:(0, \infty) \longrightarrow \mathbb{R}$ is given by $[0, \infty)$,

$$
D_{0+}^{\alpha} f(t)=\frac{1}{\Gamma(n-\alpha)}\left(\frac{d}{d t}\right)^{n} \int_{0}^{t}(t-s)^{n-\alpha-1} f(s) d s,
$$

where $n=[\alpha]+1,[\alpha]$ stands for the largest integer not greater than $\alpha$.

According to the definitions of Riemann-Liouville's derivative, the following lemmas can be achieved.

Lemma 4. For $\alpha>0$, if we assume that $u \in C[0, \infty) \cap L^{1}(0,1)$, then we have

$$
\begin{aligned}
I_{0+}^{\alpha}\left(D_{0+}^{\alpha} u(t)\right)= & u(t)+m_{1} t^{\alpha-1}+m_{2} t^{\alpha-2}+\cdots \\
& +m_{n} t^{\alpha-n},
\end{aligned}
$$

for some $m_{i} \in \mathbb{R}, i=1,2, \ldots, n$, while $n$ is the smallest integer greater than or equal to $\alpha$.

Remark 5. The following properties are useful for our discussion:

(i) As a basic example, we quote for $\lambda>-1$,

$$
D_{0+}^{\alpha} t^{\lambda}=\frac{\Gamma(\lambda+1)}{\Gamma(\lambda-\alpha+1)} t^{\lambda-\alpha}
$$

(ii) $D_{0+}^{\alpha} I_{0+}^{\alpha} u(t)=u(t)$, for $u(t) \in L^{1}(0,1)$.

For convenience, we denote

$$
\begin{aligned}
& N_{1}=\sum_{i=1}^{m} b_{i} \int_{0}^{\xi_{i}} t^{\alpha-1} d A(t), \\
& N_{2}=\sum_{i=1}^{m} d_{i} \int_{0}^{\eta_{i}} t^{\alpha-1} d B(t), \\
& M_{k}=\sum_{i=1}^{k} a_{i} \xi_{i}^{\beta-1}, \\
& E_{k}=\sum_{i=1}^{k} c_{i} \eta_{i}^{\beta-1},
\end{aligned}
$$

Besides, it should be noted that $M_{0}=E_{0}=0$. 
Lemma 6. For $h_{1}, h_{2} \in C(0,1)$, the equations

$$
\begin{aligned}
& D_{0^{+}}^{\beta}\left(\varphi_{p}\left(D_{0^{+}}^{\alpha} u(t)\right)\right)=h_{1}(t), \\
& D_{0^{+}}^{\beta}\left(\varphi_{p}\left(D_{0^{+}}^{\alpha} v(t)\right)\right)=h_{2}(t),
\end{aligned}
$$

with boundary conditions (3) and (4) have a unique solution as follows:

$$
\begin{aligned}
& u(t)=\int_{0}^{1} K_{1}(t, s) \varphi_{q}\left(\int_{0}^{1} \bar{H}(s, \tau) h_{1}(\tau) d \tau\right) d s, \\
& v(t)=\int_{0}^{1} K_{2}(t, s) \varphi_{q}\left(\int_{0}^{1} \widehat{H}(s, \tau) h_{2}(\tau) d \tau\right) d s,
\end{aligned}
$$

where

$$
K_{1}(t, s)=G_{\alpha}(t, s)+\frac{t^{\alpha-1}}{1-N_{1}} \sum_{i=1}^{m} b_{i} \int_{0}^{\xi_{i}} G_{\alpha}(t, s) d A(t),
$$

$$
K_{2}(t, s)=G_{\alpha}(t, s)+\frac{t^{\alpha-1}}{1-N_{2}} \sum_{i=1}^{m} d_{i} \int_{0}^{\eta_{i}} G_{\alpha}(t, s) d B(t),
$$

$G_{\alpha}(t, s)$

$$
= \begin{cases}\frac{1}{\Gamma(\alpha)}\left[t^{\alpha-1}(1-s)^{\alpha-1}-(t-s)^{\alpha-1}\right], & 0 \leqslant s \leqslant t \leqslant 1, \\ \frac{1}{\Gamma(\alpha)} t^{\alpha-1}(1-s)^{\alpha-1}, & 0 \leqslant t \leqslant s \leqslant 1,\end{cases}
$$$$
G_{\beta}(t, s)
$$

$$
= \begin{cases}\frac{1}{\Gamma(\beta)}\left[t^{\beta-1}(1-s)^{\beta-1}-(t-s)^{\beta-1}\right], & 0 \leqslant s \leqslant t \leqslant 1, \\ \frac{1}{\Gamma(\beta)} t^{\beta-1}(1-s)^{\beta-1}, & 0 \leqslant t \leqslant s \leqslant 1,\end{cases}
$$

and $\bar{H}(t, s)$ is a piecewise function; the expression of the $k t h$ $(k=0,1, \ldots, m)$ piece is as follows

$$
\begin{aligned}
& \bar{H}_{k}(t, s)=G_{\beta}(t, s)+\frac{t^{\beta-1}}{1-M_{m}} \sum_{i=k}^{m} a_{i} G_{\beta}\left(\xi_{i}, s\right)+\frac{M_{k}}{\left(1-M_{m}\right) \Gamma(\beta)} t^{\beta-1}(1-s)^{\beta-1} \\
& \quad= \begin{cases}\frac{1}{\Gamma(\beta)}\left[t^{\beta-1}(1-s)^{\beta-1}-(t-s)^{\beta-1}\right]+\frac{t^{\beta-1}}{\left(1-M_{m}\right) \Gamma(\beta)} \sum_{i=k+1}^{m} a_{i}\left[\xi_{i}^{\beta-1}(1-s)^{\beta-1}-\left(\xi_{i}-s\right)^{\beta-1}\right] \\
+\frac{M_{k}}{\left(1-M_{m}\right) \Gamma(\beta)} t^{\beta-1}(1-s)^{\beta-1}, & \text { for } \xi_{k} \leqslant s \leqslant t \leqslant \xi_{k+1}, \\
\frac{1}{\Gamma(\beta)} t^{\beta-1}(1-s)^{\beta-1}+\frac{t^{\beta-1}}{\left(1-M_{m}\right) \Gamma(\beta)} \sum_{i=k+1}^{m} a_{i} \xi_{i}^{\beta-1}(1-s)^{\beta-1} & \text { for } \xi_{k} \leqslant t \leqslant s \leqslant \xi_{k+1} .\end{cases}
\end{aligned}
$$

Meanwhile, $\widehat{H}(t, s)$ is also a piecewise function; the expression of $k$ th $(k=0,1 \ldots, m)$ piece is as follows

$$
\begin{aligned}
& \widehat{H}_{k}(t, s)=G_{\beta}(t, s)+\frac{t^{\beta-1}}{1-E_{m}} \sum_{i=k}^{m} c_{i} G_{\beta}\left(\eta_{i}, s\right)+\frac{E_{k}}{\left(1-E_{m}\right) \Gamma(\beta)} t^{\beta-1}(1-s)^{\beta-1} \\
& \quad= \begin{cases}\frac{1}{\Gamma(\beta)}\left[t^{\beta-1}(1-s)^{\beta-1}-(t-s)^{\beta-1}\right]+\frac{t^{\beta-1}}{\left(1-E_{m}\right) \Gamma(\beta)} \sum_{i=k+1}^{m} c_{i}\left[\eta_{i}^{\beta-1}(1-s)^{\beta-1}-\left(\eta_{i}-s\right)^{\beta-1}\right] \\
+\frac{E_{k}}{\left(1-E_{m}\right) \Gamma(\beta)} t^{\beta-1}(1-s)^{\beta-1}, & \text { for } \eta_{k} \leqslant s \leqslant t \leqslant \eta_{k+1}, \\
\frac{1}{\Gamma(\beta)} t^{\beta-1}(1-s)^{\beta-1}+\frac{t^{\beta-1}}{\left(1-E_{m}\right) \Gamma(\beta)} \sum_{i=k+1}^{m} c_{i} \eta_{i}^{\beta-1}(1-s)^{\beta-1} & \text { for } \eta_{k} \leqslant t \leqslant s \leqslant \eta_{k+1} . \\
+\frac{E_{k}}{\left(1-E_{m}\right) \Gamma(\beta)} t^{\beta-1}(1-s)^{\beta-1}, & \end{cases}
\end{aligned}
$$


Proof. We shall reduce BVP (11), (3), and (4) to two fractional differential problems. To this goal, first, by means of transformation, we convert problem (11) into

$$
\begin{aligned}
D_{0^{+}}^{\beta}\left(y_{1}(t)\right) & =h_{1}(t), \\
D_{0^{+}}^{\beta}\left(y_{2}(t)\right) & =h_{2}(t), \\
y_{1}(0) & =0, \\
y_{1}(1) & =\sum_{i=1}^{m} a_{i} y_{1}\left(\xi_{i}\right), \\
y_{2}(0) & =0, \\
y_{2}(1) & =\sum_{i=1}^{m} c_{i} y_{2}\left(\eta_{i}\right), \\
\varphi_{p}\left(D_{0^{+}}^{\alpha} u(t)\right) & =y_{1}(t), \\
\varphi_{p}\left(D_{0^{+}}^{\alpha} v(t)\right) & =y_{2}(t), \\
u(0) & =0, \\
u(1) & =\sum_{i=1}^{m} b_{i} \int_{0}^{\xi_{i}} u(t) d A(t), \\
v(0) & =0, \\
v(1) & =\sum_{i=1}^{m} d_{i} \int_{0}^{\eta_{i}} v(t) d B(t) .
\end{aligned}
$$

In view of Lemma 4, the solution of problem (17) can be written as

$$
\begin{aligned}
y_{1}(t) & =I_{0^{+}}^{\beta} h_{1}(t) \\
& =\frac{1}{\Gamma(\beta)} \int_{0}^{t}(t-s)^{\beta-1} h_{1}(s) d s+c_{1} t^{\beta-1}+c_{2} t^{\beta-2},
\end{aligned}
$$

where $c_{1}, c_{2}$ are arbitrary real constants.

By $y_{1}(0)=0$, we find $c_{2}=0$. Setting $t=1$ in (19), together with the boundary condition $y_{1}(1)=\sum_{i=1}^{m} a_{i} y_{1}\left(\xi_{i}\right)$, we have

$$
\begin{aligned}
& \frac{1}{\Gamma(\beta)} \int_{0}^{1}(1-s)^{\beta-1} h_{1}(s) d s \\
& \quad-\frac{1}{\Gamma(\beta)} \sum_{i=1}^{m} a_{i} \int_{0}^{\xi_{i}}\left(\xi_{i}-s\right)^{\beta-1} h_{1}(s) d s \\
& \quad=c_{1}\left(\sum_{i=1}^{m} a_{i} \xi_{i}^{\beta-1}-1\right) .
\end{aligned}
$$

Accordingly, we can easily find out

$$
\begin{aligned}
c_{1} & =\frac{1}{\Gamma(\beta)\left(1-M_{m}\right)}\left[\sum_{i=1}^{m} a_{i} \int_{0}^{\xi_{i}}\left(\xi_{i}-s\right)^{\beta-1} h_{1}(s) d s\right. \\
& \left.-\int_{0}^{1}(1-s)^{\beta-1} h_{1}(s) d s\right] .
\end{aligned}
$$

Combining (21) with (19), we can obtain

$$
\begin{aligned}
& y_{1}(t)=\frac{1}{\Gamma(\beta)}\left\{\int_{0}^{t}(t-s)^{\beta-1} h_{1}(s) d s\right. \\
& +\frac{t^{\beta-1}}{1-M_{m}}\left[\sum_{i=1}^{m} a_{i} \int_{0}^{\xi_{i}}\left(\xi_{i}-s\right)^{\beta-1} h_{1}(s) d s\right. \\
& \left.\left.\quad-\int_{0}^{1}(1-s)^{\beta-1} h_{1}(s) d s\right]\right\} .
\end{aligned}
$$

Next, considering the multipoint boundary conditions, we divide the domain of integration into $m+1$ pieces. For $\xi_{k} \leqslant t \leqslant \xi_{k+1}$, the unique solution of (17) can be expressed as

$$
\begin{aligned}
& y_{1}(t)=\frac{1}{\Gamma(\beta)}\left\{\int_{0}^{t}(t-s)^{\beta-1} h_{1}(s) d s\right. \\
& +\frac{t^{\beta-1}}{1-M_{m}}\left[\sum_{i=1}^{m} a_{i} \int_{0}^{\xi_{i}}\left(\xi_{i}-s\right)^{\beta-1} h_{1}(s)\right. \\
& \left.\left.-\int_{0}^{1}(1-s)^{\beta-1} h_{1}(s) d s\right]\right\}=\frac{1}{\Gamma(\beta)} \int_{0}^{\xi_{1}}\left[(t-s)^{\beta-1}\right. \\
& \left.+\frac{t^{\beta-1}}{1-M_{m}} \sum_{i=1}^{m} a_{i}\left(\xi_{i}-s\right)^{\beta-1}-\frac{t^{\beta-1}(1-s)^{\beta-1}}{1-M_{m}}\right] \\
& \cdot h_{1}(s) d s+\frac{1}{\Gamma(\beta)} \int_{\xi_{1}}^{\xi_{2}}\left[(t-s)^{\beta-1}\right. \\
& \left.+\frac{t^{\beta-1}}{1-M_{m}} \sum_{i=2}^{m} a_{i}\left(\xi_{i}-s\right)^{\beta-1}-\frac{t^{\beta-1}(1-s)^{\beta-1}}{1-M_{m}}\right] \\
& \cdot h_{1}(s) d s+\cdots+\frac{1}{\Gamma(\beta)} \int_{\xi_{k}}^{t}\left[(t-s)^{\beta-1}\right. \\
& \left.+\frac{t^{\beta-1}}{1-M_{m}} \sum_{i=k+1}^{m} a_{i}\left(\xi_{i}-s\right)^{\beta-1}-\frac{t^{\beta-1}(1-s)^{\beta-1}}{1-M_{m}}\right] \\
& \cdot h_{1}(s) d s+\frac{1}{\Gamma(\beta)} \\
& \cdot \int_{t}^{\xi_{k+1}}\left[\frac{t^{\beta-1}}{1-M_{m}} \sum_{i=k+1}^{m} a_{i}\left(\xi_{i}-s\right)^{\beta-1}\right. \\
& \left.-\frac{t^{\beta-1}(1-s)^{\beta-1}}{1-M_{m}}\right] h_{1}(s) d s+\cdots+\frac{1}{\Gamma(\beta)} \\
& \cdot \int_{\xi_{m}}^{1}\left[-\frac{t^{\beta-1}(1-s)^{\beta-1}}{1-M_{m}}\right] h_{1}(s) d s \text {. }
\end{aligned}
$$

When $\xi_{k} \leqslant s \leqslant t$, we observe that the $k$ th integrand in (23) can be taken as follows:

$$
\frac{1}{\Gamma(\beta)}\left[(t-s)^{\beta-1}+\frac{t^{\beta-1}}{1-M_{m}} \sum_{i=k+1}^{m} a_{i}\left(\xi_{i}-s\right)^{\beta-1}\right.
$$




$$
\begin{array}{lc}
\left.-\frac{t^{\beta-1}(1-s)^{\beta-1}}{1-M_{m}}\right]=\frac{1}{\Gamma(\beta)}\left\{(t-s)^{\beta-1}\right. & \sum_{i=1}^{m} b_{i} \int_{0}^{\xi_{i}} u(t) d A(t) \\
-t^{\beta-1}(1-s)^{\beta-1} & =-\frac{1}{\Gamma(\alpha)} \int_{0}^{1}(1-s)^{\alpha-1} \varphi_{q}\left(\int_{0}^{1} \bar{H}(s, \tau) h_{1}(\tau) d \tau\right) d s \\
+\frac{t^{\beta-1}}{1-M_{m}} \sum_{i=k+1}^{m} a_{i}\left[\left(\xi_{i}-s\right)^{\beta-1}-\xi_{i}^{\beta-1}(1-s)^{\beta-1}\right] & +c_{1} .
\end{array}
$$$$
-\frac{t^{\beta-1}(1-s)^{\beta-1}}{1-M_{m}}+t^{\beta-1}(1-s)^{\beta-1}
$$$$
\left.+\frac{t^{\beta-1}}{1-M_{m}} \sum_{i=k+1}^{m} a_{i} \xi_{i}^{\beta-1}(1-s)^{\beta-1}\right\}
$$$$
=\frac{1}{\Gamma(\beta)}\left\{(t-s)^{\beta-1}-t^{\beta-1}(1-s)^{\beta-1}\right.
$$$$
+\frac{t^{\beta-1}}{1-M_{m}} \sum_{i=k+1}^{m} a_{i}\left[\left(\xi_{i}-s\right)^{\beta-1}-\xi_{i}^{\beta-1}(1-s)^{\beta-1}\right]
$$$$
\left.-\frac{M_{k}}{1-M_{m}} t^{\beta-1}(1-s)^{\beta-1}\right\}=-G_{\beta}(t, s)-\frac{t^{\beta-1}}{1-M_{m}}
$$$$
\cdot \sum_{i=k+1}^{m} a_{i} G_{\beta}\left(\xi_{i}, s\right)-\frac{M_{k}}{\left(1-M_{m}\right) \Gamma(\beta)} t^{\beta-1}(1-s)^{\beta-1}
$$$$
=-\bar{H}_{k}(t, s) \text {, }
$$

Set $c_{1}$ and $c_{2}$ into (27); we find out

$$
\begin{aligned}
u(t)= & \int_{0}^{1} G_{\alpha}(t, s) \varphi_{q}\left(\int_{0}^{1} \bar{H}(s, \tau) h_{1}(\tau) d \tau\right) d s \\
& +t^{\alpha-1} \sum_{i=1}^{m} b_{i} \int_{0}^{\xi_{i}} u(t) d A(t) .
\end{aligned}
$$

Furthermore, by integrating and summing on both sides of (29), it is easy to see that

$$
\begin{aligned}
& \sum_{i=1}^{m} b_{i} \int_{0}^{\xi_{i}} u(t) d A(t)=\sum_{i=1}^{m} b_{i} \int_{0}^{\xi_{i}}\left[\int_{0}^{1} G_{\alpha}(t, s)\right. \\
& \left.\cdot \varphi_{q}\left(\int_{0}^{1} \bar{H}(s, \tau) h_{1}(\tau) d \tau\right) d s\right] d A(t) \\
& \quad+N_{1}\left(\sum_{i=1}^{m} b_{i} \int_{0}^{\xi_{i}} u(t) d A(t)\right) .
\end{aligned}
$$

Then, we get

where $\sum_{i=m+1}^{m} a_{i} G_{\beta}\left(\xi_{i}, s\right)$ is also noted by 0 .

As a result, the Green function $\bar{H}_{k}(t, s)$ is always valid and can exactly express each integrand in the above formula, for $k=0,1, \cdots m$. Thus, we can take (23) as a unified form

$$
y_{1}(t)=-\int_{0}^{1} \bar{H}(t, s) h_{1}(s) d s .
$$

$\bar{H}(t, s)$ is a piecewise function such that the form of the $k$ th piece is given by (15).

We take (25) into (18); then

$$
\varphi_{p}\left(D_{0^{+}}^{\alpha} u(t)\right)=-\int_{0}^{1} \bar{H}(t, s) h_{1}(s) d s .
$$

From Lemma 4, we have

$$
\begin{gathered}
\sum_{i=1}^{m} b_{i} \int_{0}^{\xi_{i}} u(t) d A(t)=\frac{1}{1-N_{1}} \sum_{i=1}^{m} b_{i} \int_{0}^{\xi_{i}}\left[\int_{0}^{1} G_{\alpha}(t, s)\right. \\
\left.\cdot \varphi_{q}\left(\int_{0}^{1} \bar{H}(s, \tau) h_{1}(\tau) d \tau\right) d s\right] d A(t) .
\end{gathered}
$$

Take (31) into (29); we conclude that the unique solution $u(t)$ of BVP (11), (3), and (4) is

$u(t)$

$$
\begin{aligned}
& =\int_{0}^{1}\left[G_{\alpha}(t, s)+\frac{t^{\alpha-1}}{1-N_{1}} \sum_{i=1}^{m} b_{i} \int_{0}^{\xi_{i}} G_{\alpha}(t, s) d A(t)\right] \\
& \cdot \varphi_{q}\left(\int_{0}^{1} \bar{H}(s, \tau) h_{1}(\tau) d \tau\right) d s .
\end{aligned}
$$

Analogically, the unique solution $v(t)$ of BVP (11), (3), and (4) is

$v(t)$

$$
\begin{aligned}
& =\int_{0}^{1}\left[G_{\alpha}(t, s)+\frac{t^{\alpha-1}}{1-N_{2}} \sum_{i=1}^{m} d_{i} \int_{0}^{\eta_{i}} G_{\alpha}(t, s) d B(t)\right] \\
& \cdot \varphi_{q}\left(\int_{0}^{1} \widehat{H}(s, \tau) h_{2}(\tau) d \tau\right) d s,
\end{aligned}
$$

where $\widehat{H}(t, s)$ is also a piecewise function, the $k$ th piece $\widehat{H}_{k}(t, s)$ is defined by (16), and the calculation process is similar.

This completes the proof. 
Denote

$$
\begin{gathered}
\Phi_{\alpha}(t, s) \\
= \begin{cases}t^{\alpha-1}(1-s)^{\alpha-1}-\left(t-t^{\alpha-1} s\right)^{\alpha-1}, & 0 \leqslant s \leqslant t \leqslant 1, \\
t^{\alpha-1}(1-s)^{\alpha-1}, & 0 \leqslant t \leqslant s \leqslant 1 .\end{cases} \\
\Phi_{\beta}(t, s)=\frac{1}{\Gamma(\beta)} \\
. \begin{cases}t^{\beta-1}(1-s)^{\beta-1}-\left(t-t^{\beta-1} s\right)^{\beta-1}, & 0 \leqslant s \leqslant t \leqslant 1, \\
t^{\beta-1}(1-s)^{\beta-1}, & 0 \leqslant t \leqslant s \leqslant 1 .\end{cases}
\end{gathered}
$$

Lemma 7. For $t, s \in[0,1]$ and $1<\gamma \leqslant 2$, it holds that

$$
\begin{aligned}
& t^{\gamma-1}(1-s)^{\gamma-1}-(t-s)^{\gamma-1} \\
& \quad \geqslant t^{\gamma-1}(1-s)^{\gamma-1}-\left(t-t^{\gamma-1} s\right)^{\gamma-1} \geqslant 0 .
\end{aligned}
$$

Proof. For $1<\gamma \leqslant 2$ and $0 \leqslant t \leqslant 1$, we have $t \leqslant t^{\gamma-1} \leqslant 1$, while $t^{\gamma-1}$ is a monotone increasing function. Therefore, we can infer that $t-s \leqslant t-t^{\gamma-1} s \leqslant t-t s$. For $(t-s)^{\gamma-1} \leqslant$ $\left(t-t^{\gamma-1} s\right)^{\gamma-1} \leqslant(t-t s)^{\gamma-1}$, we finally find out $(t-t s)^{\gamma-1}-(t-$ $s)^{\gamma-1} \geqslant(t-t s)^{\gamma-1}-\left(t-t^{\gamma-1} s\right)^{\gamma-1} \geqslant(t-t s)^{\gamma-1}-(t-t s)^{\gamma-1}=0$, which implies $\Phi_{\alpha}(t, s) \geqslant 0$ and $\Phi_{\beta}(t, s) \geqslant 0$, for $1<\alpha, \beta \leqslant 2$, $0 \leqslant t, s \leqslant 1$.

This completes the proof.

Lemma 8. For $t, s \in[0,1]$, the functions $G_{\alpha}(t, s), G_{\beta}(t, s)$ defined by (14) have the following properties:

(1) $G_{\alpha}(t, s)>0, G_{\beta}(t, s)>0$, for $t, s \in(0,1)$;

(2) $\Phi_{\alpha}(t, s)(1-s)^{\alpha-1} \leqslant \Gamma(\alpha) G_{\alpha}(t, s) \leqslant(1-s)^{\alpha-1}$, for $t, s \in(0,1)$;

$\Phi_{\beta}(t, s)(1-s)^{\beta-1} \leqslant G_{\beta}(t, s) \leqslant(1-s)^{\beta-1} / \Gamma(\beta)$, for $t, s \in(0,1)$.

Proof. (1) The positive of $G_{\alpha}(t, s)$ and $G_{\beta}(t, s)$ is easily checked.

(2) For $0 \leqslant s \leqslant t \leqslant 1$, we have

$$
\begin{aligned}
& \Gamma(\alpha) G_{\alpha}(t, s)=t^{\alpha-1}(1-s)^{\alpha-1}-(t-s)^{\alpha-1} \\
& \quad \leqslant t^{\alpha-1}(1-s)^{\alpha-1} \leqslant(1-s)^{\alpha-1}, \\
& \Gamma(\alpha) G_{\alpha}(t, s)=t^{\alpha-1}(1-s)^{\alpha-1}-(t-s)^{\alpha-1} \\
& \geqslant t^{\alpha-1}(1-s)^{\alpha-1}-\left(t-t^{\alpha-1} s\right)^{\alpha-1} \\
& \geqslant\left[t^{\alpha-1}(1-s)^{\alpha-1}-\left(t-t^{\alpha-1} s\right)^{\alpha-1}\right](1-s)^{\alpha-1} \\
& =\Phi_{\alpha}(t, s)(1-s)^{\alpha-1} .
\end{aligned}
$$

For $0 \leqslant t \leqslant s \leqslant 1$, we have

$$
\begin{aligned}
\Gamma(\alpha) G_{\alpha}(t, s) & =t^{\alpha-1}(1-s)^{\alpha-1} \leqslant(1-s)^{\alpha-1}, \\
\Gamma(\alpha) G_{\alpha}(t, s) & =t^{\alpha-1}(1-s)^{\alpha-1} \\
& \geqslant t^{\alpha-1}(1-s)^{\alpha-1}(1-s)^{\alpha-1} \\
& =\Phi_{\alpha}(t, s)(1-s)^{\alpha-1} .
\end{aligned}
$$

The other inequality is achieved similarly. All these complete the proof of the lemma.

For $s \in[0,1]$, denote

$$
\begin{aligned}
\varrho_{1}(s) & =\frac{1}{\left(1-\mathrm{N}_{1}\right) \Gamma(\alpha)} \sum_{i=1}^{m} b_{i} \int_{0}^{\xi_{i}} \Phi_{\alpha}(t, s) d A(t), \\
\varrho_{2}(s) & =\frac{1}{\left(1-N_{2}\right) \Gamma(\alpha)} \sum_{i=1}^{m} d_{i} \int_{0}^{\eta_{i}} \Phi_{\alpha}(t, s) d B(t), \\
\rho_{1} & =\frac{1}{\Gamma(\alpha)}\left[1+\frac{1}{1-N_{1}} \sum_{i=1}^{m} b_{i} \int_{0}^{\xi_{i}} d A(t)\right], \\
\rho_{2} & =\frac{1}{\Gamma(\beta)}\left[\frac{1}{1-M_{m}} \sum_{i=1}^{m} a_{i}+\frac{1}{1-M_{m}}\right], \\
\rho_{3} & =\frac{1}{\Gamma(\alpha)}\left[1+\frac{1}{1-N_{2}} \sum_{i=1}^{m} d_{i} \int_{0}^{\eta_{i}} d B(t)\right], \\
\rho_{4} & =\frac{1}{\Gamma(\beta)}\left[\frac{1}{1-E_{m}} \sum_{i=1}^{m} c_{i}+\frac{1}{1-E_{m}}\right],
\end{aligned}
$$

and the constant $\rho$ is given by the maximum value of $\rho_{1}, \rho_{2}$, $\rho_{3}$, and $\rho_{4}$.

Lemma 9. For $(t, s) \in[0,1] \times[0,1]$, the functions $K_{k}(t, s)(k=$ $1,2), \bar{H}(t, s)$, and $\widehat{H}(t, s)$ defined by Lemma 6 satisfy the following results:

(1) $K_{k}(t, s), \bar{H}(t, s), \widehat{H}(t, s)$ are continuous, $K_{k}(t, s) \geqslant 0$, $\bar{H}(t, s) \geqslant 0, \widehat{H}(t, s) \geqslant 0$;

(2) $\varrho_{1}(s) t^{\alpha-1}(1-s)^{\alpha-1} \leqslant K_{1}(t, s) \leqslant \rho(1-s)^{\alpha-1}$, $\varrho_{2}(s) t^{\alpha-1}(1-s)^{\alpha-1} \leqslant K_{2}(t, s) \leqslant \rho(1-s)^{\alpha-1} ;$

(3) $\Phi_{\beta}(t, s) t^{\beta-1}(1-s)^{\beta-1} \leqslant \bar{H}(t, s) \leqslant \rho(1-s)^{\beta-1}$,

$$
\Phi_{\beta}(t, s) t^{\beta-1}(1-s)^{\beta-1} \leqslant \widehat{H}(t, s) \leqslant \rho(1-s)^{\beta-1} .
$$

Proof. The nonnegativeness of $K_{k}(t, s), \bar{H}(t, s)$, and $\widehat{H}(t, s)$ is easily checked.

(2) According to the property (2) in Lemma 8, we have

$$
\begin{gathered}
K_{1}(t, s)=G_{\alpha}(t, s)+\frac{t^{\alpha-1}}{1-N_{1}} \sum_{i=1}^{m} b_{i} \int_{0}^{\xi_{i}} G_{\alpha}(t, s) d A(t) \\
\geqslant \frac{\Phi_{\alpha}(t, s)(1-s)^{\alpha-1}}{\Gamma(\alpha)}
\end{gathered}
$$




$$
\begin{gathered}
\quad+\frac{t^{\alpha-1}}{1-N_{1}} \sum_{i=1}^{m} b_{i} \int_{0}^{\xi_{i}} \frac{\Phi_{\alpha}(t, s)(1-s)^{\alpha-1}}{\Gamma(\alpha)} d A(t) \\
\geqslant \frac{t^{\alpha-1}}{1-N_{1}} \sum_{i=1}^{m} b_{i} \int_{0}^{\xi_{i}} \frac{\Phi_{\alpha}(t, s)(1-s)^{\alpha-1}}{\Gamma(\alpha)} d A(t) \\
=\varrho_{1}(s) t^{\alpha-1}(1-s)^{\alpha-1}, \\
K_{1}(t, s)=G_{\alpha}(t, s)+\frac{t^{\alpha-1}}{1-N_{1}} \sum_{i=1}^{m} b_{i} \int_{0}^{\xi_{i}} G_{\alpha}(t, s) d A(t) \\
\leqslant \frac{(1-s)^{\alpha-1}}{\Gamma(\alpha)}+\frac{t^{\alpha-1}}{1-N_{1}} \sum_{i=1}^{m} b_{i} \int_{0}^{\xi_{i}} \frac{(1-s)^{\alpha-1}}{\Gamma(\alpha)} d A(t) \\
\leqslant \frac{1}{\Gamma(\alpha)}\left(1+\frac{1}{1-N_{1}} \sum_{i=1}^{m} b_{i} \int_{0}^{\xi_{i}} d A(t)\right)(1-s)^{\alpha-1} \\
=\rho_{1}(1-s)^{\alpha-1} \leqslant \rho(1-s)^{\alpha-1} .
\end{gathered}
$$

For $k=0,1 \ldots m$, we have

$$
\begin{aligned}
& H_{k}(t, s)=G_{\beta}(t, s)+\frac{t^{\beta-1}}{1-M_{m}} \sum_{i=k+1}^{m} a_{i} G_{\beta}\left(\xi_{i}, s\right) \\
& +\frac{M_{m}}{\left(1-M_{m}\right) \Gamma(\beta)} t^{\beta-1}(1-s)^{\beta-1} \\
& \quad \leqslant \frac{1}{\Gamma(\beta)}\left(\frac{1}{1-M_{m}} \sum_{i=k+1}^{m} a_{i}+\frac{1}{1-M_{m}}\right)(1-s)^{\beta-1} \\
& \quad \leqslant \frac{1}{\Gamma(\beta)}\left(\frac{1}{1-M_{m}} \sum_{i=1}^{m} a_{i}+\frac{1}{1-M_{m}}\right)(1-s)^{\beta-1} \\
& \quad=\rho_{2}(1-s)^{\beta-1} \leqslant \rho(1-s)^{\beta-1} \cdot \\
& H_{k}(t, s)=G_{\beta}(t, s)+\frac{t^{\beta-1}}{1-M_{m}} \sum_{i=k+1}^{m} a_{i} G_{\beta}\left(\xi_{i}, s\right) \\
& \quad+\frac{M_{m}}{\left(1-M_{m}\right) \Gamma(\beta)} t^{\beta-1}(1-s)^{\beta-1} \geqslant t^{\beta-1}(1-s)^{\beta-1} \\
& \quad \cdot\left(\Phi_{\beta}(t, s)+\frac{\sum_{i=k+1}^{m} a_{i} \Phi_{\beta}\left(\xi_{i}, s\right)}{\left(1-M_{m}\right)}\right. \\
& \left.\quad+\frac{M_{m}}{\left(1-M_{m}\right) \Gamma(\beta)}\right) \geqslant t^{\beta-1}(1-s)^{\beta-1}\left(\Phi_{\beta}(t, s)\right. \\
& \left.+\frac{\sum_{i=k+1}^{m} a_{i} \Phi_{\beta}\left(\xi_{i}, s\right)}{\left(1-M_{m}\right)}\right) \geqslant t^{\beta-1}(1-s)^{\beta-1} \Phi_{\beta}(t, s) .
\end{aligned}
$$

Because of the randomicity of $k$, we have

$$
\Phi_{\beta}(t, s) t^{\beta-1}(1-s)^{\beta-1} \leqslant \bar{H}(t, s) \leqslant \rho(1-s)^{\beta-1}
$$

According to the above, from the property (2) of Lemma 8 , we easily get

$$
\begin{gathered}
\varrho_{2}(s) t^{\alpha-1}(1-s)^{\alpha-1} \leqslant K_{2}(t, s) \leqslant \rho_{3}(1-s)^{\alpha-1}, \\
\Phi_{\beta}(t, s) t^{\beta-1}(1-s)^{\beta-1} \leqslant \widehat{H}(t, s) \leqslant \rho_{4}(1-s)^{\beta-1} .
\end{gathered}
$$

This completes the proof of the lemma.

Define the space $X=\left\{u(t) \mid u(t) \in C[0,1]\right.$ and $D_{0^{+}}^{\alpha} u(t) \epsilon$ $C[0,1]\}$ is endowed with the norm $\|u\|=\max \left\{\|u\|_{0},\left\|D_{0^{+}}^{\alpha} u\right\|_{0}\right\}$, where $\|u\|_{0}=\max _{t \in[0,1]}|u(t)|,\left\|D_{0^{+}}^{\alpha} u\right\|_{0}=\max _{t \in[0,1]}\left|D_{0^{+}}^{\alpha} u(t)\right|$.

Then we introduce the product space $(X \times X,\|(u, v)\|)$ endowed with the norm $\|(u, v)\|=\max \{\|u\|,\|v\|\}$ and define a partial order $\left(\begin{array}{l}u_{1} \\ v_{1}\end{array}\right) \geqslant\left(\begin{array}{c}u_{2} \\ v_{2}\end{array}\right)$ where $u_{1}(t) \geqslant u_{2}(t), v_{1}(t) \geqslant v_{2}(t)$, for $t \in[0,1]$.

Lemma 10. $(X \times X,\|(u, v)\|)$ is a Banach space.

Proof. Let $\left\{u_{n}\right\}_{n=1}^{\infty}$ be a Cauchy sequence in the space $(X,\|\cdot\|)$. Then clearly $\left\{u_{n}\right\}_{n=1}^{\infty}$ and $\left\{D_{0^{+}}^{\alpha} u(t)\right\}_{n=1}^{\infty}$ are Cauchy sequences in the space $C[0,1]$. Therefore, $\left\{u_{n}\right\}_{n=1}^{\infty}$ and $\left\{D_{0^{+}}^{\alpha} u(t)\right\}_{n=1}^{\infty}$ converge to some $v$ and $w$ on $[0,1]$ uniformly and $v, w \in$ $C[0,1]$. Then, we need to prove $w=D_{0^{+}}^{\alpha} v$.

Note that

$$
\begin{aligned}
& \left|I_{0^{+}}^{\alpha} D_{0^{+}}^{\alpha} u_{n}(t)-I_{0^{+}}^{\alpha} w(t)\right| \\
& \quad \leqslant \frac{1}{\Gamma(\alpha)} \int_{0}^{t}(t-s)^{\alpha-1}\left|D_{0^{+}}^{\alpha} u_{n}(s)-w(s)\right| d s \\
& \quad \leqslant \frac{1}{\Gamma(\alpha+1)} \max _{s \in[0,1]}\left|D_{0^{+}}^{\alpha} u_{n}(s)-w(s)\right| .
\end{aligned}
$$

By the convergence of $\left\{D_{0^{+}}^{\alpha} u(t)\right\}_{n=1}^{\infty}$, we get

$$
\lim _{n \longrightarrow \infty} I_{0^{+}}^{\alpha} D_{0^{+}}^{\alpha} u_{n}(t)=I_{0^{+}}^{\alpha} w(t)
$$

uniformly for $t \in[0,1]$. On the other hand, by Remark 5 , one has $I_{0^{+}}^{\alpha} D_{0^{+}}^{\alpha} u_{n}(t)=u_{n}(t)+c_{1} t^{\alpha-1}+c_{2} t^{\alpha-2}$, for $t \in[0,1]$ and some $c_{1} \in R$. Furthermore, we can get

$$
\begin{aligned}
\lim _{n \longrightarrow \infty} I_{0^{+}}^{\alpha} D_{0^{+}}^{\alpha} u_{n}(t) & =\lim _{n \longrightarrow \infty} u_{n}(t)+c_{1} t^{\alpha-1}+c_{2} t^{\alpha-2} \\
& =v(t)+c_{1} t^{\alpha-1}+c_{2} t^{\alpha-2} .
\end{aligned}
$$

From (44) and (45),we obtain

$$
I_{0^{+}}^{\alpha} w(t)=v(t)+c_{1} t^{\alpha-1}+c_{2} t^{\alpha-2}, \quad \text { for } t \in[0,1] .
$$

Taking the $\alpha$-order derivative on both sides of yields, we have

$$
\begin{aligned}
& I_{0^{+}}^{\alpha} D_{0^{+}}^{\alpha} w(t)=D_{0^{+}}^{\alpha}\left[v(t)+c_{1} t^{\alpha-1}+c_{2} t^{\alpha-2}\right], \\
& \qquad \text { for } t \in[0,1] .
\end{aligned}
$$

In view of Remark 5 and Lemma 4, we find that

$$
w(t)=D_{0^{+}}^{\alpha} v(t), \quad \text { for } t \in[0,1] .
$$

Above all, we can achieve that $(X \times X,\|(u, v)\|)$ is a Banach space. This completes the proof. 
Define the cone $P \subset X \times X$ by

$$
P=\{(u, v) \in X \times X \mid u(t) \geqslant 0, v(t) \geqslant 0\} .
$$

Let the nonnegative continuous concave functional $\Psi$ on the cone $P$ be defined by

$$
\Psi((u, v))=\min \left\{\min _{\theta \leqslant t \leqslant 1-\theta}|u(t)|, \min _{\theta \leqslant t \leqslant 1-\theta}|v(t)|\right\},
$$

where $\theta$ is a positive constant, satisfying $0<\theta<1-\theta<1$.

For convenience, for $0 \leqslant s \leqslant 1$, we denote

$$
\begin{aligned}
c(s) & =\frac{1}{1-N_{1}} \sum_{i=1}^{m} b_{i} \int_{0}^{\xi_{i}} G_{\alpha}(t, s) d A(t), \\
e_{k}(s) & =\frac{1}{1-M_{m}} \sum_{i=k}^{m} a_{i} G_{\beta}\left(\xi_{i}, s\right)+\frac{M_{k}(1-s)^{\beta-1}}{\left(1-M_{m}\right) \Gamma(\beta)},
\end{aligned}
$$

where $k=0,1 \cdots m$. $T$ by

For any $(u, v) \in X \times X$, we introduce the integral operator

$$
T(u, v)(t)=\left(\begin{array}{l}
T_{1}(u, v)(t) \\
T_{2}(u, v)(t)
\end{array}\right)
$$

where

$$
\begin{aligned}
& T_{1}(u, v)(t) \\
& \quad= \int_{0}^{1} K_{1}(t, s) \varphi_{q}\left(\int_{0}^{1} \bar{H}(s, \tau)\right. \\
& \quad\left.f_{1}\left(\tau, u(\tau), v(\tau), D_{0^{+}}^{\alpha} u(\tau), D_{0^{+}}^{\alpha} v(\tau)\right) d \tau\right) d s, \\
& T_{2}(u, v)(t) \\
& \quad=\int_{0}^{1} K_{2}(t, s) \varphi_{q}\left(\int_{0}^{1} \widehat{H}(s, \tau)\right. \\
&\left.\quad \cdot f_{2}\left(\tau, u(\tau), v(\tau), D_{0^{+}}^{\alpha} u(\tau), D_{0^{+}}^{\alpha} v(\tau)\right) d \tau\right) d s .
\end{aligned}
$$

Consequently, by calculation

$$
D_{0^{+}}^{\alpha} T(u, v)(t)=\left(\begin{array}{l}
D_{0^{+}}^{\alpha} T_{1}(u, v)(t) \\
D_{0^{+}}^{\alpha} T_{2}(u, v)(t)
\end{array}\right),
$$

where

$$
\begin{aligned}
& D_{0^{+}}^{\alpha} T_{1}(u, v)(t)=-\varphi_{q}\left(\int_{0}^{1} \bar{H}(t, s)\right. \\
& \left.\cdot f_{1}\left(s, u(s), v(s), D_{0^{+}}^{\alpha} u(s), D_{0^{+}}^{\alpha} v(s)\right) d s\right), \\
& D_{0^{+}}^{\alpha} T_{2}(u, v)(t)=-\varphi_{q}\left(\int_{0}^{1} \widehat{H}(t, s)\right. \\
& \left.\quad \cdot f_{2}\left(s, u(s), v(s), D_{0^{+}}^{\alpha} u(s), D_{0^{+}}^{\alpha} v(s)\right) d s\right) .
\end{aligned}
$$

Lemma 11. $T: P \longrightarrow P$ is completely continuous.
Proof. Firstly, we prove that $T_{1}: P \longrightarrow P$. In view of the nonnegativeness and continuity of $K_{1}(t, s), \bar{H}(t, s)$ and $f_{1}(t$, $\left.u(t), v(t), D_{0^{+}}^{\alpha} u(t), D_{0^{+}}^{\alpha} v(t)\right), T_{1}$ is continuous and $T_{1}(u$, $v)(t) \geqslant 0$ for $(u, v) \in P$. Hence, $T_{1} P \subset P$.

Next, we show $T_{1}$ is uniformly bounded. Let $\Omega \subset P$ be bounded; that is, there exists a positive constant $L>0$ such that $0 \leqslant u \leqslant L, 0 \leqslant v \leqslant L,-L \leqslant D_{0^{+}}^{\alpha} u(t) \leqslant$ $L,-L \leqslant D_{0^{+}}^{\alpha} v(t) \leqslant L$ for all $(u, v) \in \Omega$. Let $A_{1}=$ $\max _{t \in[0,1]}\left|f_{1}\left(t, u(t), v(t), D_{0^{+}}^{\alpha} u(t), D_{0^{+}}^{\alpha} v(t)\right)\right|$. Then for $(u, v) \in$ $\Omega$, from the Lemma 9 , we have

$$
\begin{aligned}
& \left|T_{1}(u, v)(t)\right| \\
& \quad=\mid \int_{0}^{1} K_{1}(t, s) \varphi_{q}\left(\int_{0}^{1} \bar{H}(s, \tau)\right. \\
& \left.\quad \cdot f_{1}\left(\tau, u(\tau), v(\tau), D_{0^{+}}^{\alpha} u(\tau), D_{0^{+}}^{\alpha} v(\tau)\right) d \tau\right) d s \mid \\
& \quad \leqslant A_{1}^{q-1}\left|\int_{0}^{1} K_{1}(t, s) \varphi_{q}\left(\int_{0}^{1} \bar{H}(s, \tau) d \tau\right) d s\right| \\
& \quad \leqslant A_{1}^{q-1} \mid \int_{0}^{1} \rho(1 \\
& \quad-s)^{\alpha-1} \varphi_{q}\left(\int_{0}^{1} \rho(1-\tau)^{\beta-1} d \tau\right) d s \mid \\
& \quad=A_{1}^{q-1}\left(\frac{\rho}{\beta}\right)^{q-1} \frac{\rho}{\alpha},
\end{aligned}
$$

$$
\left|D_{0^{+}}^{\alpha} T_{1}(u, v)(t)\right|
$$$$
=\mid-\varphi_{q}\left(\int_{0}^{1} \bar{H}(t, s)\right.
$$$$
\left.\cdot f_{1}\left(s, u(s), v(s), D_{0^{+}}^{\alpha} u(s), D_{0^{+}}^{\alpha} v(s)\right) d s\right)
$$$$
\leqslant A_{1}^{q-1} \varphi_{q}\left(\int_{0}^{1} \bar{H}(t, s) d s\right)
$$$$
\leqslant A_{1}^{q-1} \varphi_{q}\left(\int_{0}^{1} \rho(1\right.
$$

$$
\left.-s)^{\beta-1} d s\right)=A_{1}^{q-1}\left(\frac{\rho}{\beta}\right)^{q-1} \text {. }
$$

Hence, $T_{1}(\Omega)$ is uniformly bounded. Then, we show $T_{1}$ is equicontinuous. Indeed, for any $(u, v) \in \Omega, t_{1}, t_{2} \in[0,1]$, $t_{1}<t_{2}$, we have

$$
\begin{aligned}
& \left|T_{1}\left(u\left(t_{2}\right), v\left(t_{2}\right)\right)-T_{1}\left(u\left(t_{1}\right), v\left(t_{1}\right)\right)\right| \\
& \left.\quad \leqslant \int_{0}^{1} \mid K_{1}\left(t_{2}, s\right)-K_{1}\left(t_{1}, s\right)\right) \mid \varphi_{q}\left(\int_{0}^{1} \bar{H}(s, \tau)\right.
\end{aligned}
$$




$$
\begin{aligned}
& \left.\cdot f_{1}\left(\tau, u(\tau), v(\tau), D_{0^{+}}^{\alpha} u(\tau), D_{0^{+}}^{\alpha} v(\tau)\right) d \tau\right) d s \\
& \leqslant A_{1}^{q-1}\left(\frac{\rho}{\beta}\right)^{q-1} \int_{0}^{1}\left|K_{1}\left(t_{2}, s\right)-K_{1}\left(t_{1}, s\right)\right| d s \\
& \leqslant A_{1}^{q-1}\left(\frac{\rho}{\beta}\right)^{q-1} \int_{0}^{1}\left(\left|G_{\alpha}\left(t_{2}, s\right)-G_{\alpha}\left(t_{1}, s\right)\right|+c(s)\right. \\
& \left.\cdot\left|t_{2}^{\alpha-1}-t_{1}^{\alpha-1}\right|\right) d s .
\end{aligned}
$$

Moreover,

$$
\begin{aligned}
& \left|D_{0^{+}}^{\alpha} T_{1}\left(u\left(t_{2}\right), v\left(t_{2}\right)\right)-D_{0^{+}}^{\alpha} T_{1}\left(u\left(t_{1}\right), v\left(t_{1}\right)\right)\right| \\
& \quad \leqslant \varphi_{q}\left(\int_{0}^{1}\left|\bar{H}\left(t_{2}, s\right)-\bar{H}\left(t_{1}, s\right)\right|\right. \\
& \left.\quad \cdot f_{1}\left(s, u(s), v(s), D_{0^{+}}^{\alpha} u(s), D_{0^{+}}^{\alpha} v(s)\right) d s\right) \\
& \quad \leqslant A_{1}^{q-1} \varphi_{q}\left(\int_{0}^{1}\left|\bar{H}\left(t_{2}, s\right)-\bar{H}\left(t_{1}, s\right)\right| d s\right) \\
& \quad \leqslant A_{1}^{q-1} \varphi_{q}\left(\int _ { 0 } ^ { 1 } \left(\left|G_{\beta}\left(t_{2}, s\right)-G_{\beta}\left(t_{1}, s\right)\right|\right.\right. \\
& \left.\left.\quad+e(s)\left|t_{2}^{\beta-1}-t_{1}^{\beta-1}\right|\right) d s\right) .
\end{aligned}
$$

Therefore, the above process implies that $T_{1}: P \longrightarrow P$ is equicontinuous for all $(u, v) \in \Omega$.

By means of the Arzela-Ascoli theorem, $T_{1}: P \longrightarrow P$ is completely continuous. Likewise, the proof of $T_{2}: P \longrightarrow$ $P$ follows the same method as $T_{1}$. Thus, $T: P \longrightarrow P$ is completely continuous.

\section{Existence Result}

For convenience, we denote

$$
\begin{aligned}
Q_{1} & =\int_{0}^{1} \varrho_{1}(s)(1-s)^{\alpha-1} \\
& \cdot \varphi_{q}\left(\int_{0}^{1} \Phi_{\beta}(s, \tau) s^{\beta-1}(1-\tau)^{\beta-1} d \tau\right) d s, \\
Q_{2} & =\int_{0}^{1} \varrho_{2}(s)(1-s)^{\alpha-1} \\
& \cdot \varphi_{q}\left(\int_{0}^{1} \Phi_{\beta}(s, \tau) s^{\beta-1}(1-\tau)^{\beta-1} d \tau\right) d s, \\
M & =\min \left\{\left(\frac{\alpha}{\rho}\right)^{q-1}\left(\frac{\beta}{\rho}\right), \frac{\beta}{\rho}\right\}, \\
N & =\max _{k=1,2}\left\{Q_{k} \theta^{\alpha-1}\right\}^{-1 /(q-1)} .
\end{aligned}
$$

Theorem 12. There exist nonnegative numbers $0 \leqslant a \leqslant b \leqslant$ $\left(\theta^{2} / \Gamma(\alpha-1)\right) c$, and $f_{k}(t, u, v, w, z)(k=1,2)$ satisfies the following conditions:
$\left(H_{1}\right) f_{k}(t, u, v, w, z) \leqslant M c^{1 /(q-1)}$, for $(t, u, v, w, z) \in[0,1] \times$ $[0, c] \times[0, c] \times[-c, c] \times[-c, c]$;

$\left(H_{2}\right) f_{k}(t, u, v, w, z)<M a^{1 /(q-1)}$, for $(t, u, v, w, z) \in[0,1] \times$ $[0, a] \times[0, a] \times[-a, a] \times[-a, a]$;

$\left(H_{3}\right) f_{k}(t, u, v, w, z)>N b^{1 /(q-1)}$, for $(t, u, v, w, z) \in[\theta, 1-$ $\theta] \times[b, c] \times[b, c] \times[-c, c] \times[-c, c]$.

Then BVP (2)-(4) has at least three positive solutions $\left(u_{1}, v_{1}\right)$, $\left(u_{2}, v_{2}\right)$, and $\left(u_{3}, v_{3}\right)$ such that

$$
\begin{aligned}
\left\|\left(u_{1}, v_{1}\right)\right\| & <a, \\
b & <\Psi\left(u_{2}, v_{2}\right)<\left\|\left(u_{2}, v_{2}\right)\right\| \leqslant c, \\
a & <\left\|\left(u_{3}, v_{3}\right)\right\| \leqslant c
\end{aligned}
$$

$$
\text { with } \Psi\left(u_{3}, v_{3}\right)<b \text {. }
$$

Proof. We will verify that conditions $\left(S_{1}\right)-\left(S_{3}\right)$ of Theorem 1 are satisfied.

Let $\bar{P}_{c}=\{(u, v) \in X \times X \mid u(t) \geqslant 0, v(t) \geqslant 0$ and $\|(u, v)\| \leqslant c\}$. We first prove that $T: \bar{P}_{c} \longrightarrow \bar{P}_{c}$. For any $(u, v) \in \bar{P}_{c}, t \in[0,1]$, we have $0 \leqslant u(t) \leqslant c, 0 \leqslant v(t) \leqslant c$, $-c \leqslant D_{0^{+}}^{\alpha} u(t) \leqslant c,-c \leqslant D_{0^{+}}^{\alpha} v(t) \leqslant c$. Consequently, combining with properties (2) and (3) of Lemma 9,

$$
\begin{aligned}
& \left|T_{1}(u, v)(t)\right| \\
& \quad=\mid \int_{0}^{1} K_{1}(t, s) \varphi_{q}\left(\int_{0}^{1} \bar{H}(s, \tau)\right. \\
& \left.\quad \cdot f_{1}\left(\tau, u(\tau), v(\tau), D_{0^{+}}^{\alpha} u(\tau), D_{0^{+}}^{\alpha} v(\tau)\right) d \tau\right) d s \mid \\
& \quad \leqslant \int_{0}^{1} \rho(1 \\
& \quad-s)^{\alpha-1} \varphi_{q}\left(\int_{0}^{1} \rho(1-\tau)^{\alpha-1} M c^{1 /(q-1)} d \tau\right) d s \\
& \quad=\int_{0}^{1} \rho(1 \\
& \quad-s)^{\alpha-1} M^{q-1} c \varphi_{q}\left(\int_{0}^{1} \rho(1-\tau)^{\alpha-1} d \tau\right) d s \\
& \quad \leqslant f_{1}\left(s, u(s), v(s), D_{0^{+}}^{\alpha} u(s), D_{0^{+}}^{\alpha} v(s)\right) d s \mid \\
& \quad=M^{q-1} c\left(\frac{\rho}{\beta}\right)^{q-1}\left(\frac{\rho}{\alpha}\right) \leqslant c, \\
& \left|D_{0^{+}}^{\alpha} T_{1}(u, v)(t)\right| \\
& \quad \mid-\varphi_{q} \int_{0}^{1} \bar{H}(t, s)
\end{aligned}
$$




$$
\begin{aligned}
& \left.-s)^{\beta-1} M c^{1 /(q-1)} d s\right)=M^{q-1} c \varphi_{q}\left(\frac{\rho}{\beta}\right) \\
& =M^{q-1} c\left(\frac{\rho}{\beta}\right)^{q-1} \leqslant c .
\end{aligned}
$$$$
=\min _{\theta \leqslant t \leqslant 1-\theta}\left|\frac{b t^{\alpha}}{\theta^{2}}\right| \geqslant \frac{b \theta^{\alpha}}{\theta^{2}}=b \theta^{\alpha-2}>b .
$$

Thus, $\left\|T_{1}(u, v)\right\| \leqslant c$ and further to get $T_{1} \bar{P}_{c} \subset \bar{P}_{c}$. Therefore $T_{1}: \bar{P}_{c} \longrightarrow \bar{P}_{c}$ is completely continuous. The proof of $T_{2}$ is the same as $T_{1}$. Thus, $T: \bar{P}_{c} \longrightarrow \bar{P}_{c}$ is completely continuous.

By the above, condition $\left(S_{2}\right)$ of Theorem 1 can be satisfied, using assumption $\left(\mathrm{H}_{2}\right)$.

Finally, in order to verify $\{(u, v) \in P(\Psi, b, c) \mid \Psi(u, v)>$ $b\} \neq \varnothing$, we set $(u, v)=\left(b t^{\alpha} / \theta^{2}, b t^{\alpha} / \theta^{2}\right)$, for $0 \leqslant t \leqslant 1$. It is easy to find that

$$
\Psi((u, v))=\min _{\theta \leqslant t \leqslant 1-\theta}\left\{\min \left|\frac{b t^{\alpha}}{\theta^{2}}\right|, \min \left|\frac{b t^{\alpha}}{\theta^{2}}\right|\right\}
$$

\section{$\Psi(T(u, v)(t))$}

$$
\begin{aligned}
& =\min \left\{\min _{\theta \leqslant t \leqslant 1-\theta}\left|T_{1}(u, v)(t)\right|, \min _{\theta \leqslant t \leqslant 1-\theta}\left|T_{2}(u, v)(t)\right|\right\} \\
& >\min \left\{N^{q-1} b \int_{0}^{1} K_{1}(t, s)\right. \\
& \left.\cdot \varphi_{q}\left(\int_{0}^{1} \Phi_{\beta}(s, \tau) s^{\beta-1}(1-\tau)^{\beta-1} d \tau\right) d s, N^{q-1} b \int_{0}^{1} K_{2}(t, s) \varphi_{q}\left(\int_{0}^{1} \Phi_{\beta}(s, \tau) s^{\beta-1}(1-\tau)^{\beta-1} d \tau\right) d s\right\} \\
& \geqslant \min \left\{N^{q-1} b \theta^{\alpha-1} \int_{0}^{1} \varrho_{1}(s)(1-s)^{\alpha-1}\right. \\
& \left.\cdot \varphi_{q}\left(\int_{0}^{1} \Phi_{\beta}(s, \tau) s^{\beta-1}(1-\tau)^{\beta-1} d \tau\right) d s, N^{q-1} b \theta^{\alpha-1} \int_{0}^{1} \varrho_{2}(s)(1-s)^{\alpha-1} \varphi_{q}\left(\int_{0}^{1} \Phi_{\beta}(s, \tau) s^{\beta-1}(1-\tau)^{\beta-1} d \tau\right) d s\right\} \\
& =\min _{k=1,2}\left\{N^{q-1} b \theta^{\alpha-1} Q_{k}\right\}=b .
\end{aligned}
$$

Hence,

$$
\begin{aligned}
& \|(u, v)\|=\max \left\{\max _{0 \leqslant t \leqslant 1}|u(t)|, \max _{0 \leqslant t \leqslant 1}|v(t)|,\right. \\
& \left.\max _{0 \leqslant t \leqslant 1}\left|D_{0^{+}}^{\alpha} u(t)\right|, \max _{0 \leqslant t \leqslant 1}\left|D_{0^{+}}^{\alpha} v(t)\right|\right\} \\
& =\max \left\{\frac{b}{\theta^{2}}, \frac{b}{\theta^{2}}, \frac{b}{\theta^{2}} \Gamma(\alpha+1), \frac{b}{\theta^{2}} \Gamma(\alpha+1)\right\}=\frac{b}{\theta^{2}} \\
& \cdot \Gamma(\alpha+1)<c .
\end{aligned}
$$

If $(u, v) \in P(\Psi, b, c)$, we have $b \leqslant u(t) \leqslant c, b \leqslant v(t) \leqslant c$, $-c \leqslant D_{0^{+}}^{\alpha} u(t) \leqslant c,-c \leqslant D_{0^{+}}^{\alpha} v(t) \leqslant c$, for all $t \in[\theta, 1-\theta]$. Then
That is, $\Psi(T(u, v)(t))>b$ for all $(u, v) \in P(\Psi, b, c)$. This shows that condition $\left(S_{1}\right)$ of Theorem 1 is also satisfied.

From the above, BVP (2)-(4) has at least three positive solutions $\left(u_{1}, v_{1}\right),\left(u_{2}, v_{2}\right)$, and $\left(u_{3}, v_{3}\right)$ such that

$$
\begin{aligned}
\left\|\left(u_{1}, v_{1}\right)\right\| & <a, \\
b & <\Psi\left(u_{2}, v_{2}\right)<\left\|\left(u_{2}, v_{2}\right)\right\| \leqslant c, \\
a & <\left\|\left(u_{3}, v_{3}\right)\right\| \leqslant c
\end{aligned}
$$

$$
\text { with } \Psi\left(u_{3}, v_{3}\right)<b \text {. }
$$

The proof is completed.

Example 13. To verify the feasibility of our conclusion, here we consider the boundary value problem of nonlinear fractional differential system as follows:

$$
\begin{aligned}
& D_{0^{+}}^{1.7}\left(\varphi_{p}\left(D_{0^{+}}^{1.2} u(t)\right)\right) \\
& \quad=f_{1}\left(t, u(t), v(t), D_{0^{+}}^{1.2} u(t), D_{0^{+}}^{1.2} v(t)\right), \\
& D_{0^{+}}^{1.7}\left(\varphi_{p}\left(D_{0^{+}}^{1.2} v(t)\right)\right) \\
& \quad=f_{2}\left(t, u(t), v(t), D_{0^{+}}^{1.2} u(t), D_{0^{+}}^{1.2} v(t)\right),
\end{aligned}
$$

with boundary conditions

$$
\begin{aligned}
& u(0)=D_{0^{+}}^{1.2} u(0)=0 \\
& u(1)=\sum_{i=1}^{2} b_{i} \int_{0}^{\xi_{i}} u(t) d A(t),
\end{aligned}
$$

$$
\varphi_{p}\left(D_{0^{+}}^{1.2} u(1)\right)=\sum_{i=1}^{2} a_{i} \varphi_{p}\left(D_{0^{+}}^{1.2} u\left(\xi_{i}\right)\right),
$$




$$
\begin{gathered}
v(0)=D_{0^{+}}^{1.2} v(0)=0 \\
v(1)=\sum_{i=1}^{2} d_{i} \int_{0}^{\eta_{i}} v(t) d B(t), \\
\varphi_{p}\left(D_{0^{+}}^{1.2} v(1)\right)=\sum_{i=1}^{2} c_{i} \varphi_{p}\left(D_{0^{+}}^{1.2} v\left(\eta_{i}\right)\right),
\end{gathered}
$$

In this model, we set $p=2$, and

$$
\begin{aligned}
& a_{1}=\frac{1}{9}, \\
& a_{2}=\frac{4}{9}, \\
& b_{1}=\frac{1}{4}, \\
& b_{2}=\frac{3}{4},
\end{aligned}
$$

$$
\begin{aligned}
& c_{1}=\frac{2}{5}, \\
& c_{2}=\frac{4}{5}, \\
& d_{1}=\frac{1}{3}, \\
& d_{2}=\frac{1}{2}, \\
& \eta_{1}=\frac{1}{2}, \\
& \eta_{2}=\frac{3}{5}, \\
& \xi_{1}=\frac{1}{3}, \\
& \xi_{2}=\frac{2}{3} .
\end{aligned}
$$

Let $A(t)=0.5 t, B(t)=0.8 t$ for $t \in[0,1]$,
It is obvious that $f_{k}(t, u, v, w, z):[0,1] \times \mathbb{R}^{+2} \times \mathbb{R}^{2} \longrightarrow$ $[0,+\infty)(k=1,2)$ is continuous. Take $\theta=0.4$; by calculation, we obtain

$$
\begin{aligned}
q & =2, \\
\rho & =12.463, \\
N_{1} & =0.220, \\
N_{2} & =0.277, \\
Q_{1} & =0.034, \\
Q_{2} & =0.044, \\
M & =0.013, \\
N & =35.327 .
\end{aligned}
$$

For $k=1,2$, set $a=5, b=10$, and $c=40000$, which satisfy $0 \leqslant a \leqslant b \leqslant\left(\theta^{2} / \Gamma(\alpha-1)\right) c$, such that

$\left(F_{1}\right) f_{k}(t, u, v, w, z) \leqslant M c$, for $(t, u, v, w, z) \in[0,1] \times$ $[0,40000]^{2} \times[-40000,40000]^{2}$;

$\left(F_{2}\right) f_{k}(t, u, v, w, z)<M a$, for $(t, u, v, w, z) \in[0,1] \times$ $[0,5]^{2} \times[-5,5]^{2}$

$\left(F_{3}\right) f_{k}(t, u, v, w, z) \geqslant N b$, for $(t, u, v, w, z) \in[0.4,0.6] \times$ $[10,40000]^{2} \times[-40000,40000]^{2}$,
Thus, all the conditions in Theorem 12 are achieved. Consequently, BVP (66) has at least three positive solutions $\left(u_{1}, v_{1}\right),\left(u_{2}, v_{2}\right)$, and $\left(u_{3}, v_{3}\right)$ such that $\left\|\left(u_{1}, v_{1}\right)\right\|<5,10<$ $\min _{t \in[0.4,0.6]}\left(u_{2}, v_{2}\right)(t)<\left\|\left(u_{2}, v_{2}\right)\right\| \leqslant 40000$, and $5<\|\left(u_{3}\right.$, $\left.v_{3}\right) \| \leqslant 40000$ with $\min _{t \in[0.4,0.6]}\left(u_{3}, v_{3}\right)(t)<10$.

\section{Data Availability}

No data were used to support this study.

\section{Conflicts of Interest}

The authors declare that they have no competing interests.

\section{Authors' Contributions}

All authors contributed equally to the manuscript, read and approved the final draft.

\section{Acknowledgments}

The work is supported by National Training Program of Innovation (No. 201910019172). The funding body plays an important role in the design of the study and analysis, calculation, and writing the manuscript. 


\section{References}

[1] Y. Wei and Z. Bai, "Solvability of some fractional boundary value problems with a convection term," Discrete Dynamics in Nature and Society, vol. 2019, Article ID 1230502, 6 pages, 2019.

[2] L. Diening, P. Lindqvist, and B. Kawohl, "Mini-workshop: the plaplacian operator and applications," Oberwolfach Reports, vol. 10, no. 1, pp. 433-482, 2013.

[3] C. Azizieh, P. Cle'ment, and E. Mitidieri, "Existence and a priori estimates for positive solutions of $p$-laplace systems," Journal of Differential Equations, vol. 184, no. 2, pp. 422-442, 2002.

[4] C. Azizieh and P. Cle'ment, "A priori estimates and continuation methods for positive solutions of $p$-laplace equations," Journal of Differential Equations, vol. 179, no. 1, pp. 213-245, 2002.

[5] X. Liu, M. Jia, and X. Xiang, "On the solvability of a fractional differential equation model involving the $p$-Laplacian operator," Computers \& Mathematics with Applications, vol. 64, no. 10, pp. 3267-3275, 2012.

[6] X. Liu, M. Jia, and W. Ge, "The method of lower and upper solutions for mixed fractional four-point boundary value problem with $p$-Laplacian operator," Applied Mathematics Letters, vol. 65, pp. 56-62, 2017.

[7] M. Feng, B. Du, and W. Ge, "Impulsive boundary value problems with integral boundary conditions and one-dimensional $p$ Laplacian," Nonlinear Analysis: Theory, Methods \& Applications, vol. 70, no. 9, pp. 3119-3126, 2009.

[8] Y. Tian, S. Sun, and Z. Bai, "Positive solutions of fractional differential equations with $p$-Laplacian," Abstract \& Applied Analysis, vol. 2017, Article ID 3187492, 8 pages, 2017.

[9] K. Sheng, W. Zhang, and Z. Bai, "Positive solutions to fractional boundary-value problems with $p$-laplacian on time scales," Boundary Value Problems, vol. 2018, article 70, 2018.

[10] T. Lv, H. Pang, and L. Cao, "Existence results for fractional differential equations with multistrip Riemann-Stieltjes integral boundary conditions," Discrete Dynamics in Nature and Society, vol. 2018, Article ID 2352789, 8 pages, 2018.

[11] J. Henderson and R. Luca, "Systems of Riemann-Liouville fractional equations with multi-point boundary conditions," Applied Mathematics and Computation, vol. 309, pp. 303-323, 2017.

[12] D. Gao and J. Li, "Existence results for impulsive fractional differential inclusions with two different Caputo fractional derivatives," Discrete Dynamics in Nature and Society, vol. 2019, Article ID 1323176, 9 pages, 2019.

[13] E. Cetin and F. S. Topal, "Existence of solutions for fractional four point boundary value problems with p-Laplacian operator," Journal of Computational Analysis and Applications, vol. 19, no. 5, pp. 892-903, 2015.

[14] Q. Li, C. Hou, L. Sun, and Z. Han, "Existence and uniqueness for a class of multi-term fractional differential equations," Applied Mathematics and Computation, vol. 53, no. 1-2, pp. 383-395, 2017.

[15] K. Shah and R. A. Khan, "Existence and uniqueness results to a coupled system of fractional order boundary value problems by topological degree theory," Numerical Functional Analysis and Optimization, vol. 37, no. 7, pp. 887-899, 2016.

[16] B. Di and H. Pang, "Existence results for the fractional differential equations with multi-strip integral boundary conditions," Applied Mathematics and Computation, vol. 59, no. 1-2, pp. 1-19, 2019.

[17] C. Gao, Z. Gao, and H. Pang, "Existence criteria of solutions for a fractional nonlocal boundary value problem and degeneration to corresponding integer-order case," Advances in Difference Equations, vol. 2018, article 408, 2018.

[18] Y. Sun and M. Zhao, "Positive solutions for a class of fractional differential equations with integral boundary conditions," Applied Mathematics Letters, vol. 34, pp. 17-21, 2014.

[19] M. Cui, Y. Zhu, and H. Pang, "Existence and uniqueness results for a coupled fractional order systems with the multistrip and multi-point mixed boundary conditions," Advances in Difference Equations, vol. 2017, article 224, 2017.

[20] M. Feng, P. Li, and S. Sun, "Symmetric positive solutions for fourth-order $n$-dimensional $m$-Laplace systems," Boundary Value Problems, vol. 2018, article 63, 2018.

[21] X. Zhang, "Exact interval of parameter and two infinite families of positive solutions for a $n$th order impulsive singular equation," Journal of Computational and Applied Mathematics, vol. 330, pp. 896-908, 2018.

[22] M. Feng, "New results of coupled system of $k$ Hessian equations," Applied Mathematics Letters, vol. 94, pp. 196-203, 2019.

[23] X. Liu and M. Jia, "Solvability and numerical simulations for BVPs of fractional coupled systems involving left and right fractional derivatives," Applied Mathematics and Computation, vol. 353, pp. 230-242, 2019.

[24] X. Liu and M. Jia, "The method of lower and upper solutions for the general boundary value problems of fractional differential equations with $p$-Laplacian," Advances in Difference Equations, vol. 2018, article 28, 2018. 


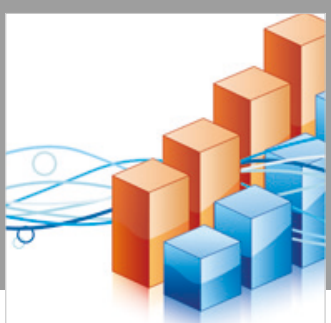

Advances in

Operations Research

\section{-n-m}
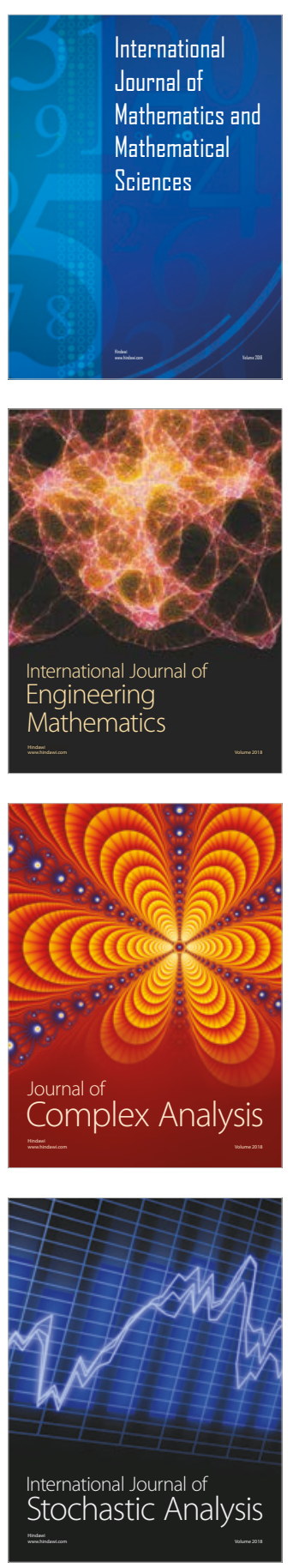
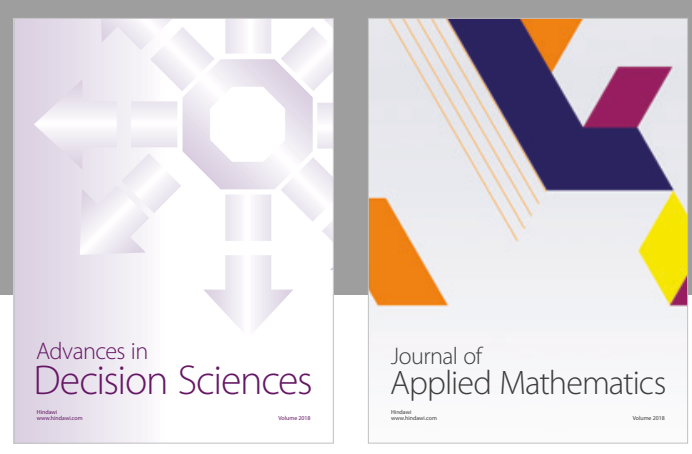

Journal of

Applied Mathematics
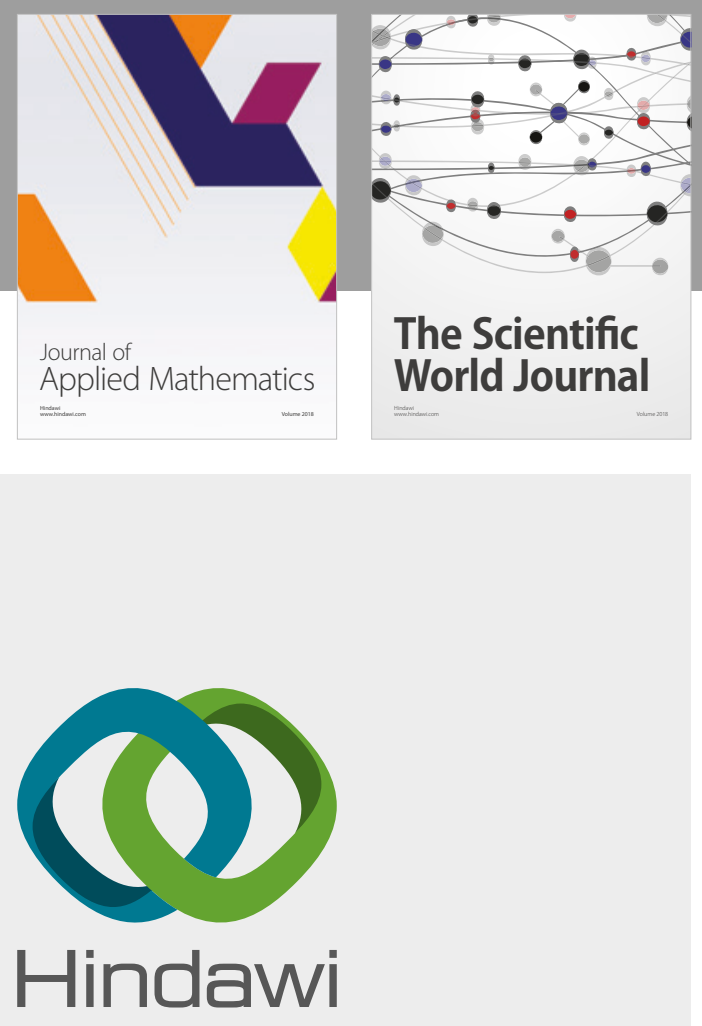

Submit your manuscripts at

www.hindawi.com

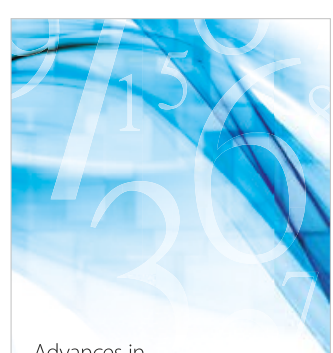

Advances in
Numerical Analysis
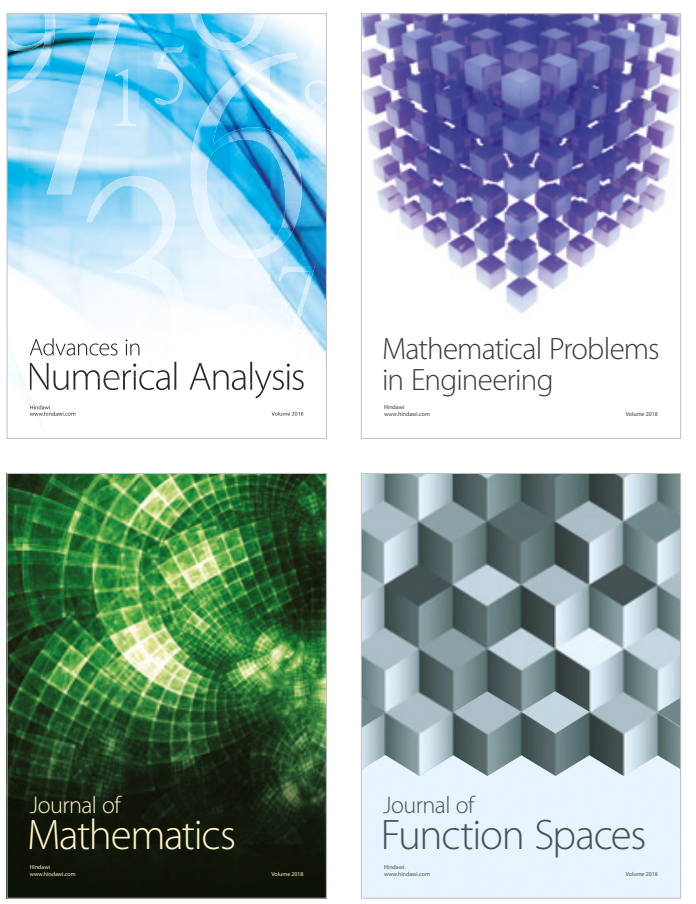

Mathematical Problems in Engineering

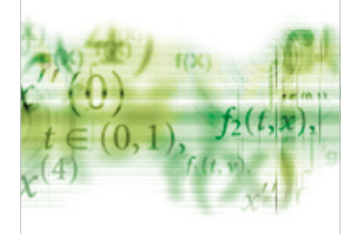

International Journal of

Differential Equations

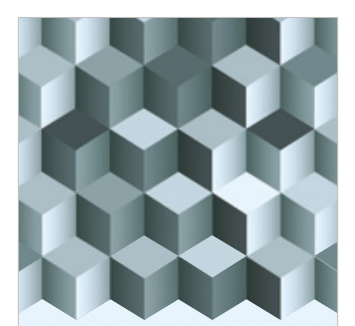

Journal of

Function Spaces

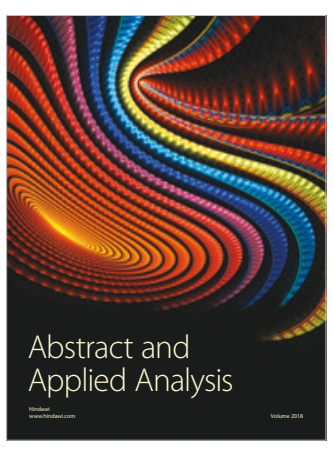

The Scientific

World Journal

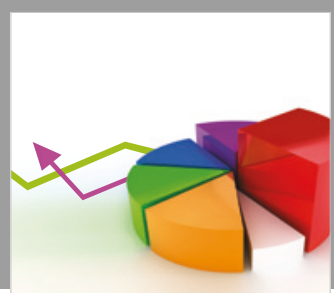

Journal of

Probability and Statistics
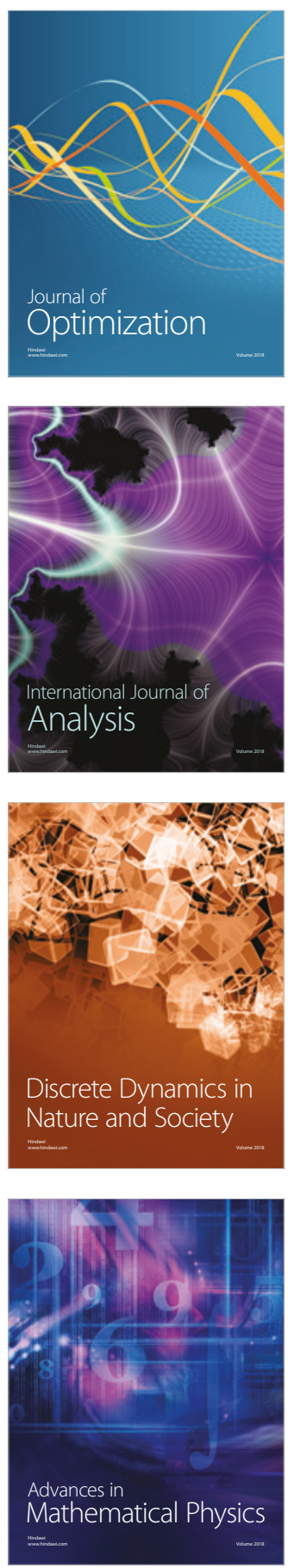